\title{
FABRICATION AND TESTING OF A LOW-COST WIND TURBINE BLADE USING BAMBOO REINFORCED RECYCLED PLASTIC
}

\author{
P. Y. Andoh ${ }^{1^{*}}$, C. K. K. Sekyere ${ }^{2}$, G. K. K. Ayetor ${ }^{3}$, M. N. Sackey ${ }^{4}$ \\ Department of Mechanical Engineering, Kwame Nkrumah University of Science and \\ Technology, Kumasi, Ghana ${ }^{1,2,3,4}$ \\ pyandoh.coe@gmail.com
}

Received : 01 April 2021, Revised: 09 June 2021, Accepted : 09 June 2021

*Coresponding Author

\begin{abstract}
Ghana's energy generation mix has about $65 \%$ thermal component which comes with increased generating cost and substantial air pollution. Wind energy has been considered as a viable sustainable energy alternative in Ghana with extensive feasibility studies being done over the years to map out suitable zones for future projects. This study presents the fabrication of wind turbine blades using bamboo fibre reinforced with recycled plastic and its assessment for lowcost wind energy generation. Bamboo fibre extracted from raw bamboo was combined with processed recycled High-Density Polyethylene (HDPE) to form the composite material in a proportion of $25 \%$ bamboo-fibre and $75 \%$ recycled HDPE. Conventional turbine blades and turbine blades made of the composite material were installed at the test site one at a time and tested under similar operating conditions. Results showed that the unit cost of energy for the bamboo-composite blade was $0.016 \mathrm{GHC} / \mathrm{kWh}$ whereas that for conventional blade was 0.018 GHC/kWh. Result obtained from Stress and deformation analysis showed that the convention wind turbine blade has slightly better strength than bamboo-plastic composite blade. The study showed that the reinforced bamboo-HDPE composite material possesses appreciable mechanical properties and also offers a cheaper alternative for wind turbine blade fabrication.
\end{abstract}

Keywords : Wind Turbine Blade, Wind Energy, Sustainable Energy, Bamboo Fibre, Recycled Plastic.

\section{Introduction}

Wind energy is now being considered by many countries as a viable sustainable energy alternative with the potential to produce clean energy and help accelerate the global agenda to mitigate the impact of climate change. It must be noted that significant efforts have been made by Developed countries in the development and harnessing of wind energy through both on shore and offshore wind turbine technology for the purpose of producing electricity. However, not much progress has been made in developing countries with regards to the use of wind energy as a cleaner production technology. Most developing nations have achieved noticeable advancements with the use of solar PV technology as compared with wind turbine technology. However, evidence shows that wind energy generation is being explored in developing countries with a lot of feasibility studies being done to identify potential sites for such projects. Typically, a typical utility-scale wind turbine can produce 1.5 to 4.0 million kWh annually and operates $70-85 \%$ of the time (Zhang et al., 2012). Global wind energy production set a record in 2011, reaching $239 \mathrm{GW}, 3 \%$ of total electricity production. It is predicted that by 2020 it will increase to $10 \%$ of global electricity production. The American Wind Energy Association reported in 2010 that production of small horizontal-axis wind turbines would increase rapidly in the future due to huge demand. For the classification of horizontal-axis wind turbines, there is no fixed standard. The National Renewable Energy Laboratory (NREL) in the US defines wind turbines whose rated power are not greater than $100 \mathrm{~kW}$ and whose diameter is no more than $19 \mathrm{~m}$ as small wind turbines (Hand et al., 2012). Wind power devices are now used to produce electricity, and commonly termed wind turbines.

The orientation of the shaft and rotational axis determines the first classification of the wind turbine. A turbine with a shaft mounted horizontally parallel to the ground is known as a horizontal axis wind turbine or (HAWT) whereas a vertical axis wind turbine (VAWT) has its 
shaft normal to the ground. The two configurations have instantly distinguishable rotor designs, each with its own favourable characteristics (Hau, 2006). The discontinued mainstream development of the VAWT can be attributed to a low tip speed ratio and difficulty in controlling rotor speed. Difficulties in the starting of vertical turbines have also hampered development, believed until recently to be incapable of self-starting (Dominy, 2007). However, the VAWT requires no additional mechanism to face the wind and heavy generator equipment can be mounted on the ground, thus reducing tower loads. Therefore, the VAWT is not completely disregarded for future development. A novel V-shaped VAWT rotor design is currently under investigation which exploits these favourable attributes (Holdsworth, 2009).

A focus is now being made on the HAWT due to its dominance in the wind turbine industry. HAWT are very sensitive to changes in blade profile and design. The efficiency of a turbine can be increased with higher tip speeds (Gasch \& Twele, 2002), although the increase is not significant when considering some penalties such as increased noise, aerodynamic and centrifugal stress. A higher tip speed demands reduced chord widths leading to narrow blade profiles. This can lead to reduced material usage and lower production costs. Although an increase in centrifugal and aerodynamic forces is associated with higher tip speeds. The increased forces signify that difficulties exist with maintaining structural integrity and preventing blade failure. As the tip speed increases the aerodynamics of the blade design become increasingly critical. A blade which is designed for high relative wind speeds develops minimal torque at lower speeds. This results in a higher cut in speed (Duquette \& Visser, 2003) and difficulty self-starting. A noise increase is also associated with increasing tip speeds as noise increases approximately proportionately to the sixth power (Gasch \& Twele, 2002; Oerlemans et al., 2006). Modern HAWT generally utilize a tip speed ratio of nine to ten for two bladed rotors and six to nine for three-bladed rotors $(\mathrm{Hu}$, 2006). This has been found to produce efficient conversion of wind kinetic energy into electrical power (Burton, 2011; Hau, 2006).

The ideal plan form of a HAWT rotor blade is defined using the BEM method by calculating the chord length according to Betz limit, local air velocities and aerofoil lift. Several theories exist for calculating the optimum chord length which range in complexity (Chattot, 2003; Duquette \& Visser, 2003; Gasch \& Twele, 2002; Hau, 2006). For blades with tip speed ratios of six to nine utilizing aerofoil sections with negligible drag and tip losses, Betz's momentum theory gives a good approximation (Hau, 2006). In instances of low tip speeds, high drag aerofoil sections and blade sections around the hub, this method could be considered inaccurate. In such cases, wake and drag losses should be accounted for (Chattot, 2003; Gasch \& Twele, 2002).

According to a report from the National Renewable Energy Laboratory, wind turbines are predominantly made of steel (71-79\% of total turbine mass); fiberglass, resin or plastic (11-16\%); iron or cast iron (5-17\%); copper (1\%); and aluminium (0-2\%). The stiffness of composites is determined by the stiffness of fibers and their volume content. Typically, E-glass fibers are used as main reinforcement in the composites. A typical glass/epoxy composites for wind turbine blades contain up to $75 \%$ glass by weight. This increases the stiffness, tensile and compression strength. A promising composite material is glass fiber with modified compositions like S-glass and R-glass. Other glass fibers developed by Owens Corning are ECRGLAS, Advantex and WindStrand (Ashwill, 2009). Due to high material cost and the desire to achieve better performance, several researchers have made efforts to develop new composite materials which have the desired mechanical properties suitable for wind turbine blade fabrication. This study seeks to develop a composite material out of Recycled High-Density Polyethylene and bamboo fibre for the fabrication of wind turbine blades.

Evidence shows that conventional petroleum-based packaging plastics are versatile, relatively economical, easily processed, have excellent properties and are resistant to degradation. High-density polyethylene (HDPE), low-density polyethylene (LDPE) and polypropylene (PP) are some of those synthetic plastics which are widely used as packaging films (carrier bags), mulching films and for similar other purposes. HDPE contains carbon and hydrogen as backbone elements and has little branching; it also has stronger intermolecular forces and tensile strength than LDPE (Vasudha et al., 2018). Physically, it is harder, more opaque, can withstand somewhat higher temperatures and therefore, has extensive industrial and day-to-day applications. However, with the expanding range of products, the consumption of these plastics is increasing incessantly, 
but the plastic waste gets accumulated abundantly in the" environment causing "white pollution", plastic waste management problems, perceived garbage crisis, shortage of landfills and many other environmental and sanitation challenges in developing countries including Ghana.

Bamboo has comparative ecological qualities (Liese \& Kohl, 2015). Most prominently, it is very sustainable. Its quality is equivalent to that of wood. Therefore, it makes an engaging upand-comer as an auxiliary material. It has been utilized broadly in family unit items and stretched out to modern applications because of advances in handling innovation and expanded market requests. The utilization ranges from family utilities, for example, compartments, chopsticks, woven mats, angling shafts, cricket boxes, crafted works, seats, and so on; to building applications, for example, flooring, roof, dividers, windows, entryways, wall, lodging 51 rooftops, brackets, rafters and purlins. Bamboo's breadth, thickness, and between nodal length have a perceptibly evaluated structure while the fiber dispersion shows an infinitesimally reviewed design, which lead to great properties of bamboo (Jansen, 2012).

Shao \& Wang (2018) studied mechanical Properties of the Vulgaris bamboo and established that longitudinal flexible modulus and elasticity of Vulgaris bamboo have clear reliance on outspread position. Tractable modulus of versatility was found to have a mean increment over all layers by $12.8 \%$ from 1.3 to $4 \mathrm{~m}$.

Widiastuti (2016) worked and presented a report focusing on the use of bamboo composite matrix for producing wind turbine blades and their performance compared with glass fiber turbine blades with their workable viewpoint. It was concluded that bamboo fulfilled the requirements of constructing a turbine blade with high performance associated with sustainable development.

Bamboo is one of the natural materials which is being intensively studied to substitute this synthetic fibre since they possess higher strength to weight ratio (Fu et al., 2012). The potential of bamboo in polymer composite is very important today due to its fibre extraction, structural variation, thermal properties, chemical modification, and mechanical properties that make it very suitable for use in the composite industry (Mounika et al., 2012; Takagi \& Fuji, 2014). Bamboo can be used in composites as a natural fibre reinforcement in polymer matrices which are petroleum based. Such composites are also called Bamboo Fibre Reinforced Polymer (BFRP) (Abdul et al., 2012).

Bamboo fibres have provided some good response in the production of materials that are recyclable, biodegradable, and sustainable (Adamu et al., 2018). Products of composites made from bamboo have several positive advantages such as dimensional stability, longevity, weather resistant, high impact resistant, low maintenance, environmental-friendliness and low flame spread (Rao et al., 2009). In the production of safety products, it has been observed that curtains produced from bamboo fibres can absorb electromagnetic radiations (X-ray, infrared) at various wavelengths, making it exclusively less harmful to the human body when compared with other curtains produced from various materials ( $\mathrm{Li}$ et al. 2012).

In order to have a turbine blade that is environmentally friendly and cost-effective, this work looks at the fabrication and testing of low-cost wind turbine blades using bamboo plastic composite which have good mechanical properties for wind turbine blade fabrication..

\section{Materials and Methods}

\subsection{Materials}

Materials used for this research are high density polyethylene (HDPE) and green bamboo (Bambusoideae). The properties of the bamboo and the used plastics that were used are tabulated and presented in Table 1.

Table 1 - Properties of the samples used.

\begin{tabular}{lcccc}
\hline Material & $\begin{array}{c}\text { Young's Modulus } \\
(\mathrm{GPa})\end{array}$ & $\begin{array}{c}\text { Ultimate Tensile } \\
\text { Strength }(\mathrm{MPa})\end{array}$ & $\begin{array}{c}\text { Fracture Toughness } \\
(\mathrm{MPa})\end{array}$ & $\begin{array}{c}\text { Percentage Elongatior } \\
(\%)\end{array}$ \\
\hline Green Bamboo & $1.2 \pm 0.065$ & $100.3 \pm 11.28$ & $109.23 \pm 5.69$ & - \\
Recycled HDPE & $0.0663 \pm 0.010$ & $16.80 \pm 0.26$ & - & $322.68 \pm 33.37$ \\
\hline
\end{tabular}

Source: (Andoh et al., 2016 ; Tawiah et al., 2016) 


\subsection{Development of the Wind Turbine Blade}

A 10-metre bamboo was harvested from a bamboo farm using a cutlass and cut into pieces of 1-m length. These pieces were then split into strips of $0.25 \mathrm{~m}$ long and immersed in a solution of $400 \mathrm{ml}$ Hydrochloric acid (HCL) which was diluted with 20 litres of water in a black container as shown in Figure 2.1 for 48 hours. This was done to make it soft and also to reduce the lignin content of the bamboo to prevent it from breaking during the fibre extraction.

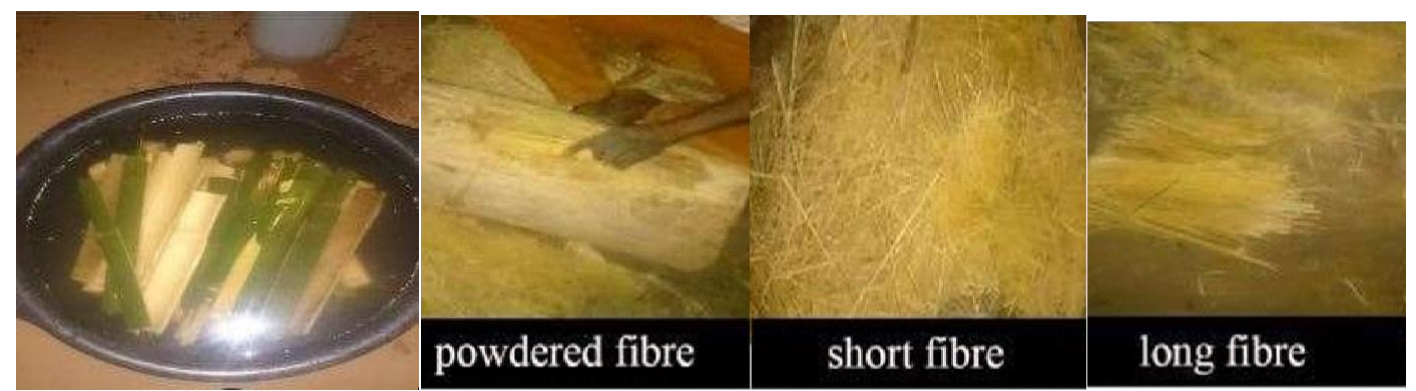

Fig 1. Processing of bamboo fibre and the sample obtained

The wet slabs were removed from the black container and beaten with a wooden mallet to obtain the bamboo fibre as shown in figure 1. Three types of fibres were obtained after the extraction process which are long and short fibres and the powdered form as shown in Figure 1. The used high-density polyethylene (HDPE) was collected, washed and cleaned, and allowed to dry. The clean HDPE were cut into pieces using knives and scissors as shown in Figure 2.

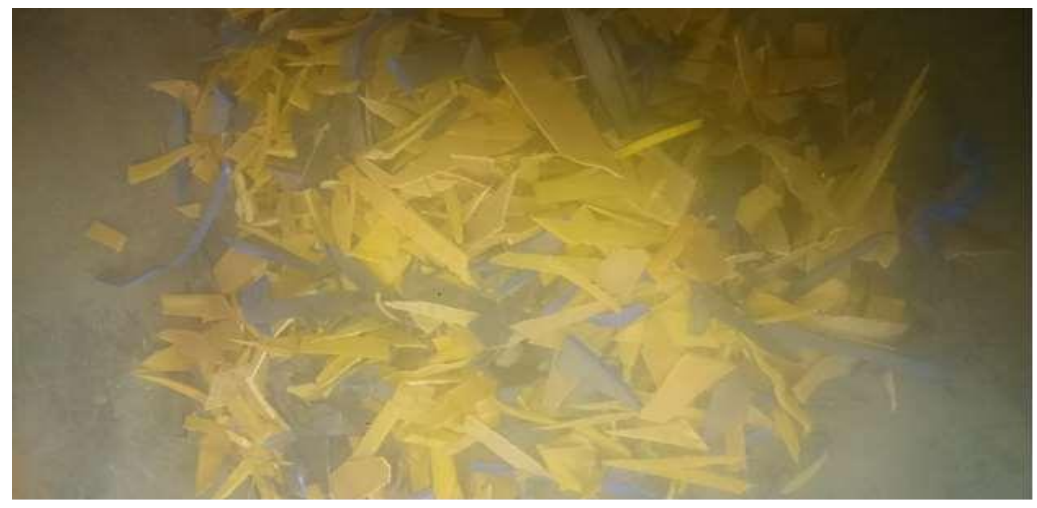

Fig 2. Cut pieces of the waste HDPE

The bamboo fibre and the recycled plastic composite were formed using the method of casting and hand-laying techniques. A $30 \mathrm{~g}$ of high-density polyethylene was placed in a source pan and fire was set on it in the saucepan. The source pan was then placed on a coal pot to accelerate the melting process. Molten HDPE was obtained within 30 to 40 minutes as shown in Figure 3.

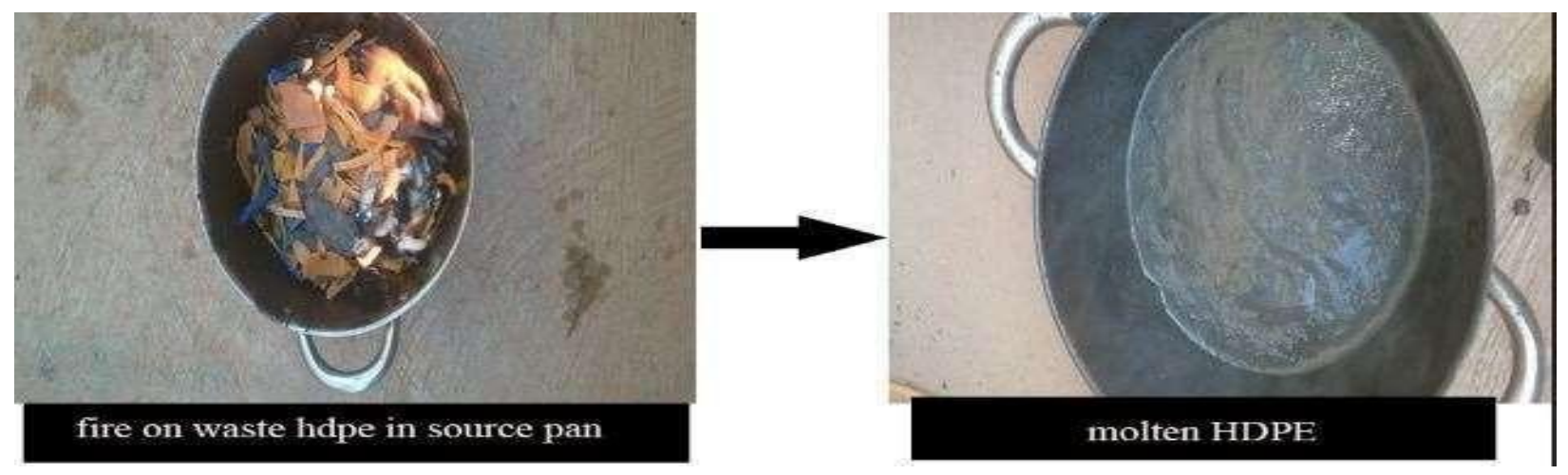

Fig 3. Melting of the shredded HDPE 
A white paper was placed in the mould and $0.5 \mathrm{~g}$ of powdered bamboo fibre was then spread on the paper in the mould. About $20 \%$ of molten HDPE was poured into the mould and mixed with the powdered fibre in the mould until a uniform mixture was obtained. $9.5 \mathrm{~g}$ of bamboo fibre was then arranged horizontally in the mould on top of the formed mixture. The remaining $80 \%$ of the molten HDPE was poured into the mould on the horizontally arranged bamboo fibre. The mould was covered and a dead weight of $10 \mathrm{~kg}$ was placed on the mould cover so as to remove any porosity or air bubbles and allowed for solidification.

Each slab was then marked out in a division of five segments for cutting out the shape of the wind turbine blade. All rough edges of the blade are smoothened to attain the set of blades as shown in Figure 4

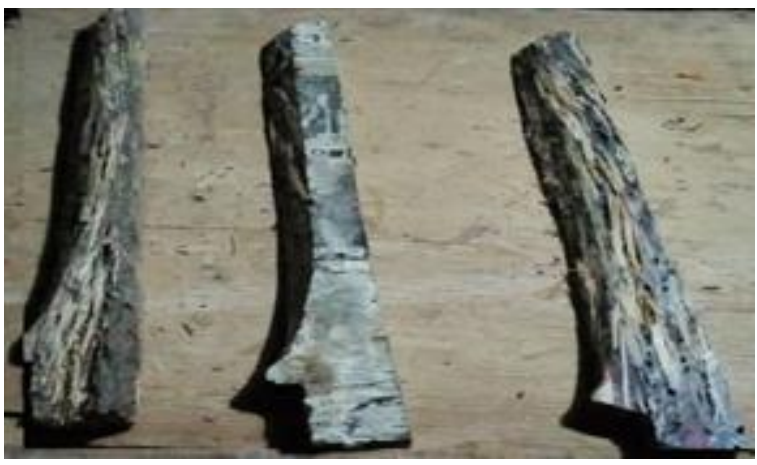

Fig. 4 Final shaped blade

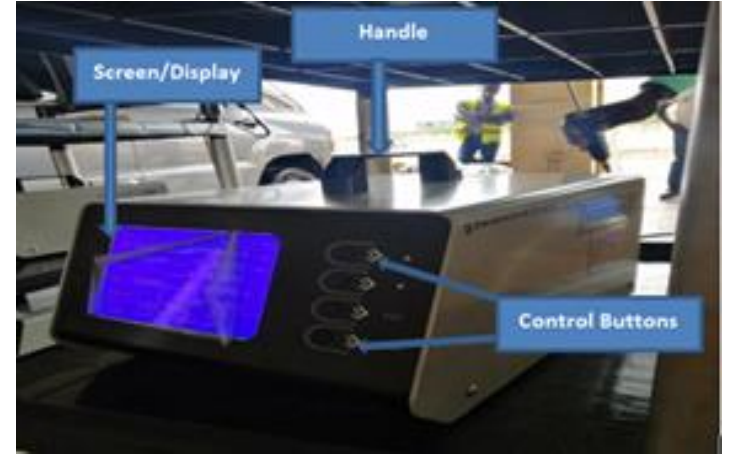

Fig. 5 Inverter with Integral turbine monitor

Finally, attachment holes to the turbine hub were drilled at the root end of the blade. The blades were manually cut without automation and the aerofoils were drafted from the glass fibre blade.

\subsection{Testing of the blade}

The field test was conducted at Ada (Latitude: 5 45' 59.99" N and Longitude: 0 36' 59.99" E) near a solar farm where a small. On the test day, the average wind speed was 2 to $2.5 \mathrm{~m} / \mathrm{s}$ from the south west, average air density of $1.225 \mathrm{~kg} / \mathrm{m}^{3}$ and the average temperature was $29^{\circ} \mathrm{C}$ with a relative humidity between $80 \%$ and $86 \%$ and was mainly sunny throughout the day. Hourly temperature measure was confirmed by using the industrial CAT make thermometer. The topography of the area was a flat plain land with mangrove vegetation and structure close to the site was 25 meters from the test location. The Raum energy wind turbine was used for the field test for both the conventional turbine blade and the bamboo plastic composite blade with the technical details shown in Table 2. Figure 5 shows the inverter with the integral turbine control monitor. 
Table 2 - Specification of the wind turbine

\begin{tabular}{lll}
\hline \multicolumn{1}{c}{ Parameters } & Bamboo Plastic Composite & Blade Material \\
\hline Power output & $3.75 \mathrm{~kW}$ & Conventional turbine blade \\
Blade Type & 3 blades down wind & $3.75 \mathrm{~kW}$ \\
Generator & Gearless, brush permanent magnet & 3 blades down wind \\
Swept Area & $0.79 \mathrm{~m}^{2}$ & Gearless, brush permanent magnet \\
Life expectancy-turbine & 20 years & $0.79 \mathrm{~m}^{2}$ \\
Inverter type & Grid tie & 20 years \\
Power Factor & 0.99 & Grid tie \\
Control System & Kaca Blue Planet 1502x & 0.99 \\
Turbine Height & 10 meters & Kaca Blue Planet 1502x \\
Anemometer & NKT 40C 3-cap & 10 meters \\
\hline
\end{tabular}

Both the conventional fibre glass composite turbine blade and the bamboo composite were installed at the test site at a height of 10 meters and tested under similar operating conditions. Data was collected for both types of blade by monitoring the following parameters: power output, wind velocity, blade rpm and the tip to speed ratio using the turbine control monitor and the values obtained were recorded.

\subsection{Finite Element Analysis}

The ANSYS software was used to model both types of wind turbine blades. Figure 6 shows the flow chart for the finite element analysis. The ANSYS modeling activity was done in three processes, which are the preprocessor, the solution and the post processor. The FEM was provided with dimensions of the composite blade and its airfoil was selected. Along the length of the composite blade there is a twist in which the value is $15^{\circ} \mathrm{C}$

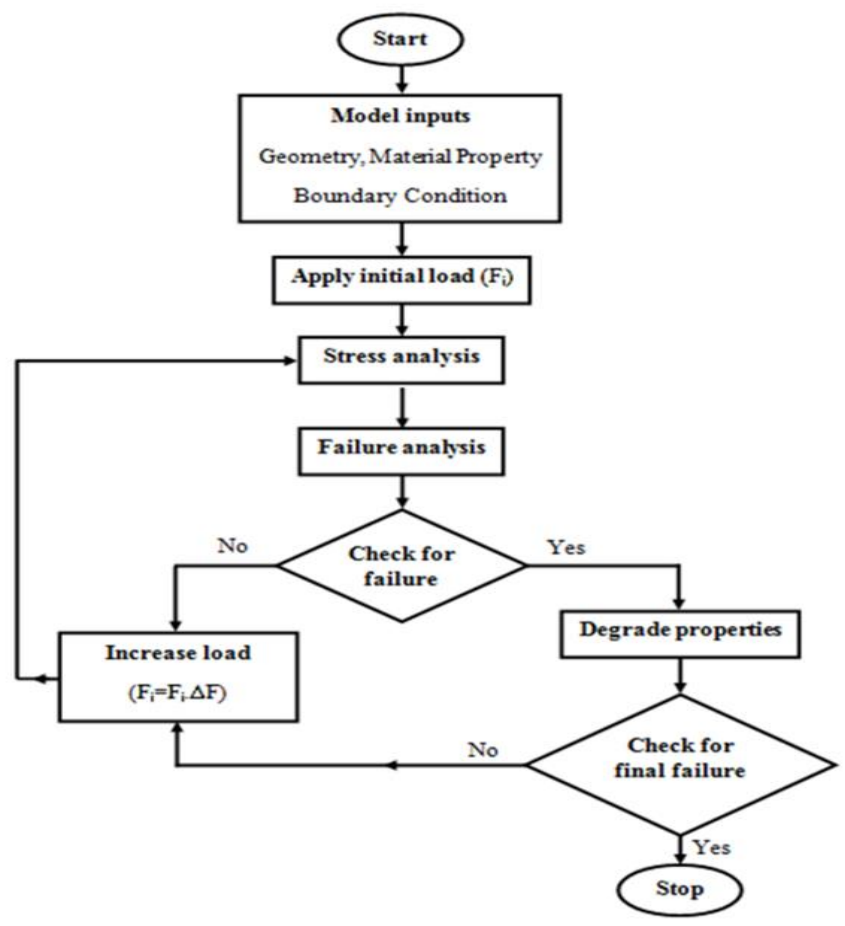

Fig 6. Flow Diagram of a Finite Element Analysis 


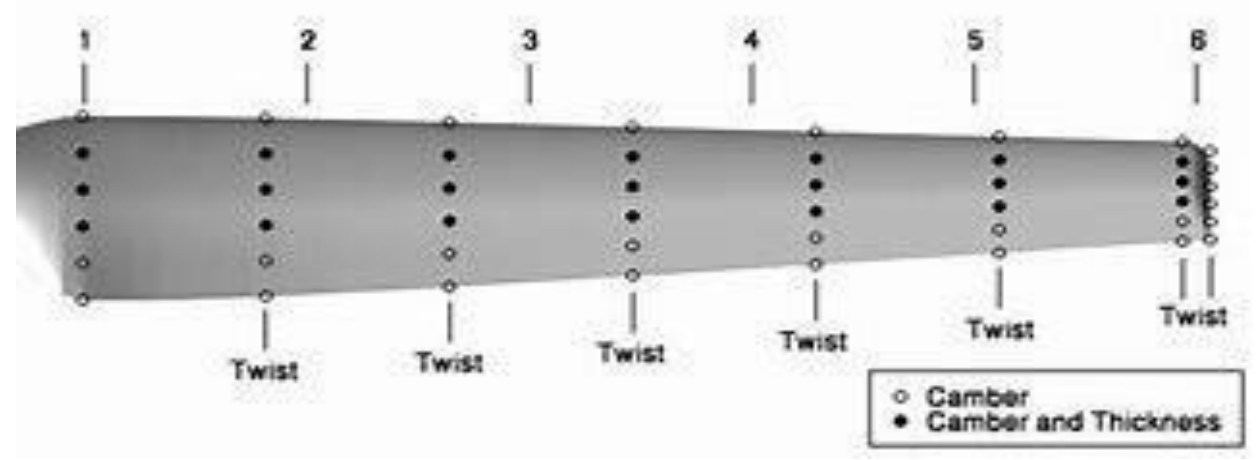

Fig 7. Shell99

Shell99 (Figure 7) was used in ANSYS in order to have the layup of the blade structure. To do the simulation, the blade was divided into five regions. The optimal or convergence analysis of the blade was done, and the structure had 25,823 elements. Wind loads have greater influence on structural design and as the blade will be placed in a wind prone zone, the effect of the wind must be considered. Wind pressure reactions on the blade differ according to their surface or airfoils. In simulation, the blade pressure side which is the Intrados was set to a pressure of $+0.85 \mathrm{kPa}$ and the lower pressure side which is the Extrados was under pressure of $-0.85 \mathrm{kPa}$. Pressure at the trailing end of the blade was set to $+0.1 \mathrm{kPa}$ due to turbulence effect. Bending loads techniques were simulated where the wind loads were applied in the direction of the blade towards and away from the extrados which is the suction side. It was also directed towards and away from the leading edge. The Buckling effect was done by positioning the blade in a vertical position and the effect of the blade on the structure when the blade turns at $90^{\circ}$. The force of gravity was increased at the tip towards the root of the blade.

\section{Results and Discussions}

\subsection{Building of the Bamboo plastic composite blade}

In the fabrication of the rotor blade, the efficiency of the blades aerodynamic performance was critical and extra caution was taken in shaping the blade as the lift force which produces the power to turn the blade is a very important element at the initial stage. Improper design of the airfoils according to the design will cause the blade not to have a good sweep. For structured stability, the root of the blade needed more attention in shaping it. There are three sections namely; the root, mid span and the tip.

The tip sweep ratio is an important element, so a good tip design will aid in capturing the wind. From the root, the aero foils were carved at twist angles towards the tip at an angle of $0^{\circ}$ to about $15^{\circ}$. Cutting the outer shape of the blade with the band saw was easy but the most difficult part is the formation of the twist for the formation of the airfoils. Great care was taken in order not to shape out airfoil thickness (twist angle). Also, transition of the root and the first aerofoil profile is part of the blade that carries most of its load. Because of its relatively low velocity drive, it causes the aerodynamic leading end for the blade to have a large chord length. This causes the blade profile to be large at the rotor end. The mid span of the blade is also very significant in the blade fabrication so it should be shaped with the modern blade profile to increase its aerodynamic lift to drag ratio by using the thinnest aerofoil section of the structure. The geometry of the tip was designed to reduce wind energy capture losses and noise. Good tip design also maximizes the aerodynamic lift to drag ratio. The fabricated blade made of the composite was formed to the above profile and this was achieved by using the shape and profile of the glass fibre blade. At the end of the fabrication, the two blades had the same dimensions and shape, but the only difference is the material type. 


\subsection{The Life Cycle of the Blade}

From the S-N curve (Figure 8), the life for the bamboo plastic composite is 106 cycles and this is true for all the fatigue tests done from week one to week three. The conventional glass fibre turbine blade has an average life of between 107 and 108 cycles which gives it a better life compared to the bamboo plastic composite. In comparison, the conventional turbine blade will last longer than the bamboo plastic composite as it has a higher life cycle.

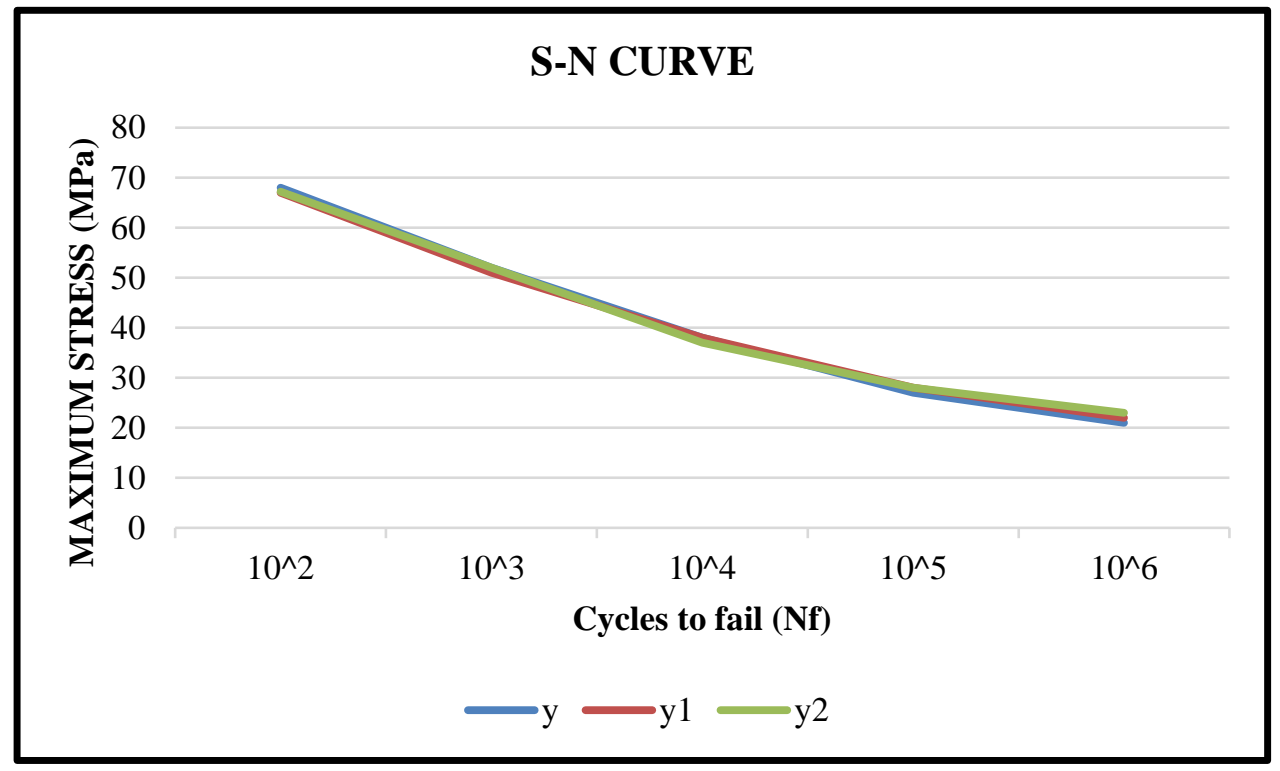

Fig 8. S-N Curve of the Bamboo plastic composite blade

From the energy production equation;

$E=C p \rho \frac{A}{2} V^{3} t$

Eqn. 1

where $C p$ is the coefficient of power, $\rho$ is the density of the air, $\mathrm{A}$ is the swept area, $\mathrm{t}$ is the lifetime and $\mathrm{V}$ is the wind velocity. Comparing the energy production of the two blades, under uniform conditions, only one factor can influence the energy production results of the blades and this factor is the life cycle of the blade. The lifetime of conventional glass fiber blades which is between $10^{7}$ and $10^{8}$ is higher so it will last longer than the bamboo plastic composite turbine blades. From this view, the conventional turbine blades energy output would be higher than the bamboo plastic composite turbine blades. The designed wind speed for the bamboo-HDPE composite turbine is $4 \mathrm{~m} / \mathrm{s}$ but it was observed that the bamboo-RHDPE composite blade was able to work at a wind speed as low as $0.8 \mathrm{~m} / \mathrm{s}$. The digital parameter display reader from the turbine gave a display of the blade rpm, wind speed, power coefficient and the voltage output from the turbine. From these parameters, the wind power is obtained using the equation;

$$
P=\frac{1}{2} \rho A V^{3}
$$

Eqn. 2

where $\rho=$ the density of air $\left(1.225 \mathrm{~kg} / \mathrm{m}^{3}, \mathrm{v}\right.$ is the velocity of the Air, A $=$ the swept area $=\pi r^{2}$. Table 3 and 4 give the energy generation at $15 \mathrm{sec}$ intervals. 
Table 3 - Power output and Energy generation for bamboo plastic composite blade

\begin{tabular}{|c|c|c|c|c|c|}
\hline Time (s) & $\begin{array}{l}\text { Wind Speed } \\
\quad(\mathrm{rpm})\end{array}$ & $\begin{array}{c}\text { Blade Speed } \\
(\mathrm{rpm})\end{array}$ & $\begin{array}{l}\text { Velocity } \\
(\mathrm{m} / \mathrm{s})\end{array}$ & $\begin{array}{l}\text { Power } \\
\text { (W) }\end{array}$ & $\begin{array}{c}\text { Energy } \\
(\mathrm{J})\end{array}$ \\
\hline 15 & 2 & 21.5 & 1.29 & 0.88 & 210.46 \\
\hline 30 & 2.2 & 22.3 & 1.34 & 0.98 & 234.84 \\
\hline 45 & 2.1 & 22.7 & 1.36 & 1.03 & 247.7 \\
\hline 60 & 2.3 & 23.4 & 1.4 & 1.13 & 271.33 \\
\hline 75 & 2.2 & 22 & 1.32 & 0.94 & 225.49 \\
\hline 90 & 2.05 & 23 & 1.38 & 1.07 & 257.66 \\
\hline 105 & 2 & 21.7 & 1.3 & 0.9 & 216.39 \\
\hline 120 & 2.15 & 22.1 & 1.33 & 0.95 & 228.58 \\
\hline 135 & 2.2 & 23 & 1.38 & 1.07 & 257.66 \\
\hline 150 & 2.3 & 22.8 & 1.37 & 1.05 & 250.99 \\
\hline \multicolumn{4}{|c|}{ Mean } & 1 & 240.11 \\
\hline \multicolumn{4}{|c|}{ Standard Deviation } & 0.08 & 19.95 \\
\hline
\end{tabular}

Table 3 gives the power output from the Bamboo-plastic composite blade and the energy generation at 15 seconds interval respectively. The standard deviation of the power and energy are $0.08 \mathrm{~W}$ and $19.95 \mathrm{~J}$ respectively. Also, the mean value for Power is $1.00 \mathrm{Watts}$ and the average energy is 240.11 Joules. Table 3.2 gives the power output from the conventional wind turbine blade and the corresponding energy generation at 15 seconds intervals. The standard deviation of the power and energy are $0.13 \mathrm{~W}$ and $31.11 \mathrm{~J}$ respectively. Also, the mean value for Power is 1.25 Watts and the average energy is 300.35 Joules. Hence, it can be observed that the conventional wind turbine blade gives $60.24 \mathrm{~W}$ energy higher than the Bamboo-Recycled HDPE composite with respect to mean energy values computed over the tests period. However, the standard deviation recorded for the bamboo composite material (0.08) was much lower than that recorded for the conventional blade. 
Table 4 - Power output and Energy generation for conventional wind turbine blade

\begin{tabular}{llllll}
\hline Time $(\mathrm{s})$ & $\begin{array}{l}\text { Wind Speed } \\
(\mathrm{rpm})\end{array}$ & Wind Speed (rpm) & Velocity $(\mathrm{m} / \mathrm{s})$ & Power $(\mathrm{W})$ & $\begin{array}{l}\text { Energy } \\
(\mathrm{J})\end{array}$ \\
\hline 15 & 2 & 23.5 & 1.41 & 1.15 & 274.83 \\
30 & 2.2 & 24.2 & 1.45 & 1.25 & 300.12 \\
45 & 2.1 & 23.7 & 1.42 & 1.17 & 281.9 \\
60 & 2.3 & 25.2 & 1.51 & 1.41 & 338.89 \\
75 & 2.2 & 24.6 & 1.48 & 1.31 & 315.25 \\
90 & 2.05 & 23.2 & 1.39 & 1.1 & 264.44 \\
105 & 2 & 23 & 1.38 & 1.07 & 257.66 \\
120 & 2.15 & 24.1 & 1.45 & 1.24 & 296.42 \\
135 & 2.2 & 24.9 & 1.49 & 1.36 & 326.93 \\
150 & 2.3 & 25.4 & 1.52 & 1.45 & 347.02 \\
Mean & & & & 1.25 & 300.35 \\
Standard Deviation & & & 0.13 & 31.11 \\
\hline
\end{tabular}

\subsection{Cost Benefit Analysis}

Table 3 shows the cost breakdown for both types of wind turbine blade used for the purpose of the study. It can be observed that the Bamboo-HDPE composite wind turbine blade cost GHC 3,550 less than the cost of purchasing and importing the conventional fibre class composite wind turbine bade.

Table 5 - Cost breakdown of the Bamboo Plastic Composite and the Glass conventional turbine Blade

\begin{tabular}{lclr}
\hline \multicolumn{2}{c}{ Bamboo fibre plastic blade } & \multicolumn{2}{c}{ Conventional turbine blade } \\
Items & Cost GHC & Items & Cost GHC \\
\hline Bamboo & 50.00 & Cost, Insurance, freight & 2640.00 \\
Plastic flakes & 40.00 & Import Duty & 660.00 \\
Heating cost & 10.00 & Clearing Charges & 850.00 \\
Metal Mould & 70.00 & & \\
Production cost & 50.00 & & \\
Transport cost & 30.00 & & 3850.00 \\
Miscellaneous & 50.00 & & \\
Total Cost & 300.00 & & \\
\hline
\end{tabular}

The cost for producing the bamboo reinforced plastic blade is GHC 300.00 for a set of three blades, while the cost of purchase and importation of the conventional Glass fibre blade cost GHC $3,850.00$. So, in effect, there will be a marginal savings of GHC 3,550.00 which makes it appealing in economic terms. In effect, the cost of importing 1 set of conventional glass fibre blades can be used to produce three sets of the bamboo plastic composite blade. Secondly, the importation of the conventional wind turbine blades can also be affected by foreign currency exchange rates making the bamboo reinforced plastic blade a preferred choice. For the energy generation, the average blade speed and wind power was used since the maximum and minimum values were obtained and their means computed with the results presented in Table 6 . 
Table 6 - Cost per kWh for the bamboo plastic composite blade and the glass fibre blade

\begin{tabular}{lcc}
\hline Items & $\begin{array}{c}\text { Bamboo-plastic } \\
\text { composite blade }\end{array}$ & $\begin{array}{c}\text { Convention wind } \\
\text { turbine Blade }\end{array}$ \\
\hline Life $(\mathrm{H})$ & 76.5 & 723 \\
Generated energy $(\mathrm{J})$ & 240.11 & 300.35 \\
Total Energy Generated for life span $(\mathrm{kJ})$ & 18.36 & 216.9 \\
GHC Cost per Blade & 300 & 3850 \\
GHC Cost per kWh & 0.016 & 0.018 \\
\hline
\end{tabular}

From the energy generated, the cost per $\mathrm{kWh}$ for the bamboo plastic composite blade is lower than that of the glass fibre composite, making bamboo plastic composite cheaper and readily available. Looking at their lifespan, the bamboo composite blade has a life span which is one tenth that of the glass fibre blade but in terms of cost, the bamboo plastic composite blade is about thirteen times cheaper than the glass fibre making it a preferred choice.

\subsection{Finite Element Analysis}

The static analysis for both the bamboo fibre composite blade and the glass fibre wind turbine had its stress and deformation analyzed using ANSYS with their results showing different stress and deformation levels in Table 7.

Table 7 - Simulation results of equivalent (von-Mises) stress and deformation

\begin{tabular}{lcc}
\hline Location & Von-Mises Stress (MPa) & Total Deformation (mm) \\
\hline Glass Fibre & 0 & 3.74 \\
Bamboo Plastic composite & 16.52 & 3.41 \\
\hline
\end{tabular}

The bamboo plastic composite had a maximum deformation of $3.41 \mathrm{~mm}$ and that of the glass fibre was $3.71 \mathrm{~mm}$ (see Figure 9). The difference in their maximum deformation is small and this makes the bamboo plastic composite blade acceptable for application as a turbine blade. Too much deformation will cause the blade not to have a good sweep angle.

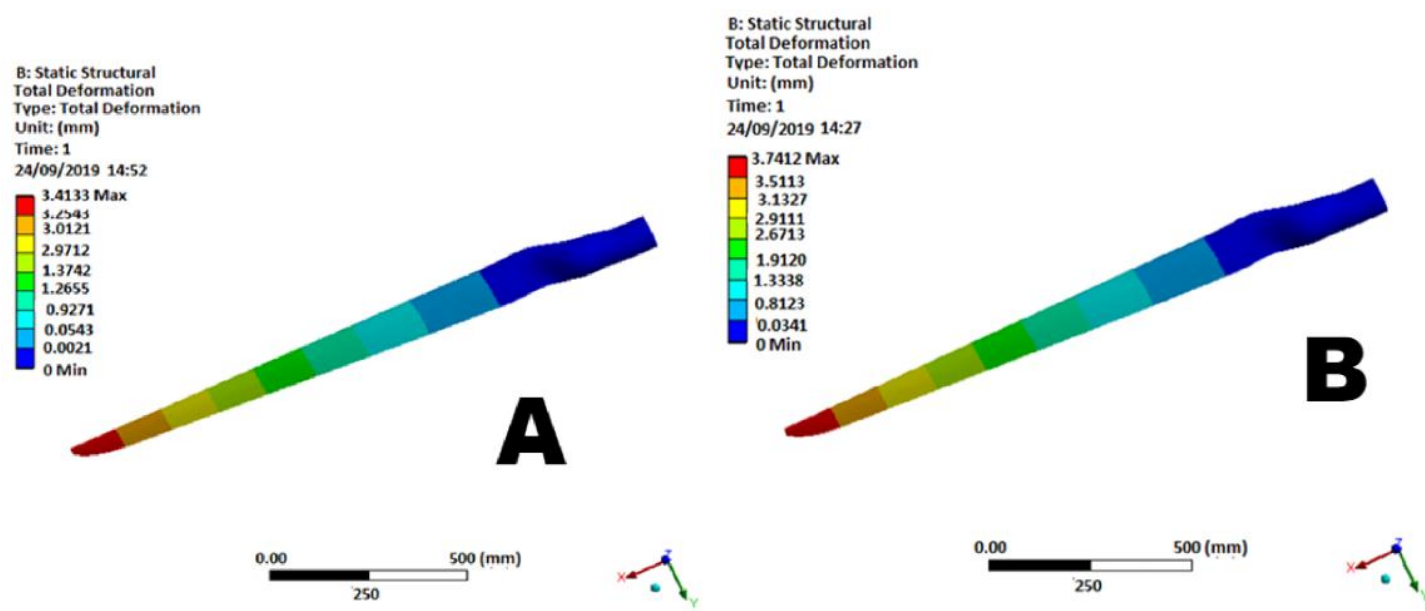

Fig 9. Deformation of (A) Bamboo fibre reinforced plastic turbine blade \& (B) Glass fibre turbine blade

The stress at different points of the blade was considered with the aim of controlling safety factors against the applied loads. The maximum and critical values were the most important parameters looked at to have a safe blade operation. Looking at the analyzed blades, the stress was in the area of the root and this was influenced by the aerodynamic forces and maximum bending moments. Figure 10 shows the maximum and minimum stresses for both the bamboo fibre composite and the glass fibre composite. 


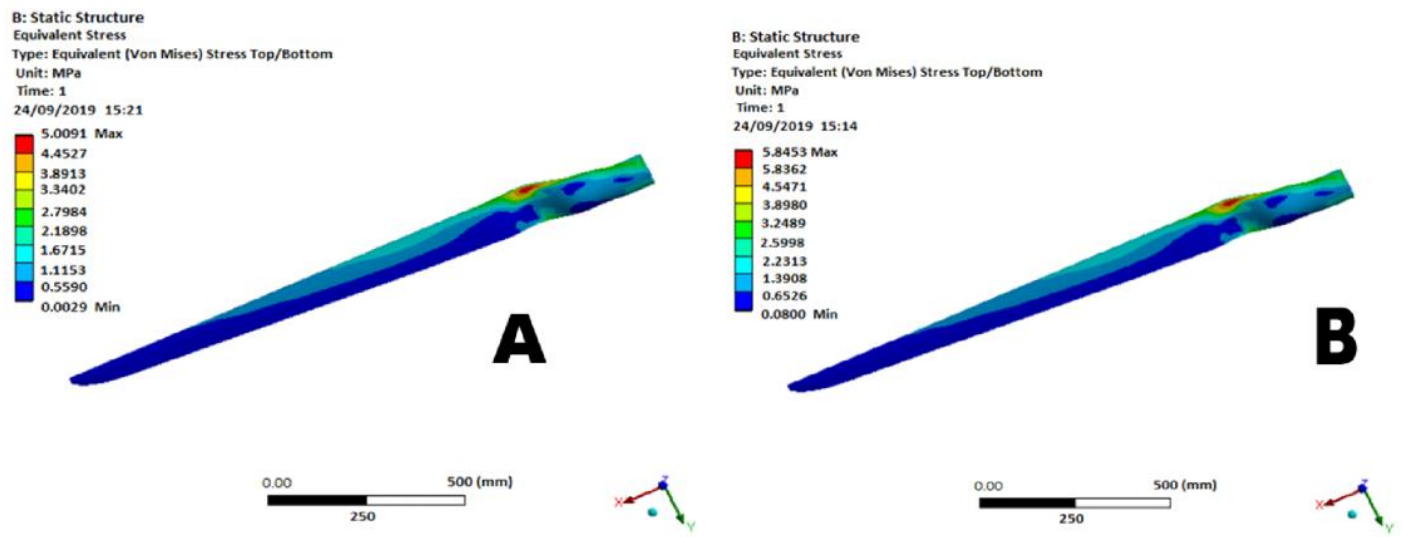

Fig 10. Von-Mises stress of (A) Bamboo fibre reinforced plastic turbine blade \& (B) Glass fibre turbine blade

Both blades were modeled for the stresses and the results showed that there was a lower stress on the Bamboo fibre reinforced plastic blade which was 5.01 MPa and higher stress on the Glass fibre of value 5.84 MPa. The Maximum yield stress of the bamboo fibre composite is 5.28 $\mathrm{MPa}$, with its Von Mises stress is lower than the yield stress of the material thereby making the composite material safer to be used as a turbine blade. The stress distribution and deformation are similar for both the glass fibre and bamboo fibre reinforced plastic. The maximum deformation always occurs at the tip of the blade since it's free to move and the maximum stress occurs at the root area because it's fixed at that point. The values of stress and deformation for both blades vary, and this is because a blade with higher weight value leads a higher centrifugal force from the equation.

Table 8 - ANSYS results in the XYZ plane

\begin{tabular}{lcc}
\hline Distribution & Maximum & Minimum \\
\hline Directional deformation in X-axis (mm) & 3.41 & 0 \\
Directional deformation in Y-axis (mm) & 0.68 & -30.5 \\
Directional deformation in Z- axis (mm) & 12.55 & -6.21 \\
Normal Stress in X- axis (MPa) & 5.01 & 0.01 \\
Normal Stress in Y-axis (MPa) & 1.74 & -2.19 \\
Normal Stress in Z- axis (Mpa) & 5.67 & -5.67 \\
Shear Stress in XY- axis (MPa) & 2.41 & -1.89 \\
Shear Stress in YZ- axis (MPa) & 0.86 & -0.92 \\
Shear Stress in XZ- axis (MPa) & 1.97 & -1.12 \\
\hline
\end{tabular}

From Table 8, it can be observed that the directional deformation in $\mathrm{X}$ axis has a maximum value of $3.14 \mathrm{~mm}$ and a minimum of $0 \mathrm{~mm}$, the directional deformation at $\mathrm{Y}$ axis in maximum case $0.68 \mathrm{~mm}$ and a minimum of $-30.5 \mathrm{~mm}$ and deformation in the $\mathrm{Z}$ axis has a maximum of $12.55 \mathrm{~mm}$ and minimum of $-6.21 \mathrm{~mm}$. Also, the deformations about $\mathrm{x}$ and $\mathrm{y}$ axis which was maximum at tip of the blade and minimum at the root end and was similarly for the $\mathrm{z}$ axis where the maximum and minimum deformations occurred at the same location. The normal stresses in $\mathrm{X}$ and $\mathrm{Z}$ directions at maximum and minimum was recorded at middle location but for normal stresses in Y direction it was at the right edge of the blade. The shear stress in the XY, YZ, XZ planes also had values recorded as shown in Table 6 . The shear stress in the XY plane at maximum of $2.41 \mathrm{~mm}$ and a minimum of $-1.89 \mathrm{~mm}$. The shear stress of both maximum and minimum occurred at the same location. The shear stress in the ZY plane at maximum of $0.86 \mathrm{~mm}$ and minimum of $0.86 \mathrm{~mm}$. Both shear stresses took place at the same location and at the right-side edge position. For the XZ plane, the shear stress is at maximum of $1.97 \mathrm{~mm}$ and minimum at -1 . $12 \mathrm{~mm}$ took place at the same location. 


\section{Conclusion and Recommendation}

The objective of this project was to fabricate and test a wind turbine blade using bamboo reinforced with recycled plastic for low-cost wind energy generation applications. This was achieved by building a blade from bamboo reinforced recycled plastic composite, testing the blade to determine its performance on the wind turbine, doing cost benefit analysis and modelling the blade to determine its stress and deformation behaviour.

It was established that the locally fabricated bamboo plastic composite blade is the best option with regards to the initial investment since the cost of importing one set of the conventional glass fibre composite turbine blade is thirteen times that of the bamboo plastic composite made locally. Secondly, considering their lifespans, the glass fibre composite has between $107-108$ life cycles while that of the bamboo plastic composite is 106 cycles. Comparing their $\mathrm{kWh}$ cost, the bamboo plastic composite cost GHC 0.016 per $\mathrm{kWh}$ as compared to GHC 0.018 per $\mathrm{kWh}$ for the conventional glass fibre composite turbine bade, making the energy produced from the bamboo plastic blade less expensive. Taking all the above factors into consideration, it can be observed that it is more cost effective to invest in the bamboo plastic composite turbine blade with a lower initial cost and a lower cost per $\mathrm{kWh}$ of energy produced.

However, considering the relatively low life cycle/life span of the fabricated BambooRecycled HDPE composite material, there is the need to further develop the composite material by varying the compositions of Bamboo fibre and Recycled HDPE contents until a desired proportion is achieved which has the potential to give a life span equivalent to that of the conventional wind turbine blade with equivalent material strength. The extremely shorter life span is seen as a limitation of the study. However, the emphasis of this research is that composite materials produced from readily available local materials such as Bamboo and Recycled HDPE should be developed for use in wind turbine blade fabrication since they have the potential to give relatively lower energy unit cost as compared to the conventional turbine blade materials. Hence, barring the lower life cycle of the fabricated composite material, it is seen as a more attractive option because of its comparatively lower manufacturing cost.

As a future scope, the fluid structure interaction study can be done to find the formation of eddies, rotor wakes and the flow of wind around the blade. Testing and analysis of the blade can be done by varying conditions such as in humidity and high and cold temperature zones. The finite element modelling should also be used to approximate solution methods for continuous systems of equations. Also, the area of improving the material structure and characteristics can be looked since it's a huge research field to improve the effectiveness of the blade.

\section{References}

Ashwill, T.D. (2009). Materials and Innovations for Large Blade Structures: Research Opportunities in Wind Energy Technology. 50th AIAA Structures, Structural Dynamics, \& Materials Conference Palm Springs, May, 2009 (AIAA-2009-2407), American Institute of Aeronautics and Astronautics, pp 1- 20.

Burton, T. (2011). Wind Energy Handbook; John Wiley \& Sons Ltd.: Chichester, UK.

Chattot, J. J. (2003). Optimization of wind turbines using helicoidal vortex model. J. Sol. Energy Eng., 125(4), 418-424.

Dominy, R., Lunt, P., Bickerdyke, A., \& Dominy, J. (2007). Self-starting capability of a Darrieus turbine. Proceedings of the Institution of Mechanical Engineers, Part A: Journal of Power and Energy, 221(1), 111-120.

Duquette, M. M., \& Visser, K. D. (2003). Numerical implications of solidity and blade number on rotor performance of horizontal-axis wind turbines. J. Sol. Energy Eng., 125(4), 425432.

Fu Jiajia, Zhang X, Yu Chongwen, Guebitz Georg M, Cavaco-paulo Artur (2012). Bioprocessing of bamboo materials. Fibres Text Eastern Europe 2012; 1:13-9.

Gasch, R., \& Twele, J. (Eds.). (2011). Wind power plants: fundamentals, design, construction and operation. Springer Science \& Business Media.

Hand, M. M., Baldwin, S., DeMeo, E., Reilly, J. M., Mai, T., Arent, D., ... \& Sandor, D. (2012). Renewable electricity futures study. volume 1. exploration of high-penetration 
renewable electricity futures (No. NREL/TP-6A20-52409-1). National Renewable Energy Lab.(NREL), Golden, CO (United States).

Hau, E. (2006). Wind Turbines-Fundamentals, Technologies, Application, Economics, 2nd ed.; Springer: Berlin, Germany, 2006.

Holdsworth, B. (2009). Green Light for Unique NOVA Offshore Wind Turbine. Available online: http://www.reinforcedplastics.com (accessed on 8 May 2012).

Janssen, J. J. (2012). Mechanical Properties of Bamboo. Berlin: Springer Science \& Business Media.

Khalil, H. A., Bhat, I. U. H., Jawaid, M., Zaidon, A., Hermawan, D., \& Hadi, Y. S. (2012). Bamboo fibre reinforced biocomposites: A review. Materials \& Design, 42, 353-368.

Liese, W., \& Kohl, M. (2015). Bamboo. The plant and its uses. Switzerland.

Mounika, M., Ramaniah, K., Prasad, A. R., Rao, K. M., \& Reddy, K. H. C. (2012). Thermal conductivity characterization of bamboo fiber reinforced polyester composite. J. Mater. Environ. Sci, 3(6), 1109-1116.

Muhammad, A., Rahman, M. R., Hamdan, S., \& Sanaullah, K. (2019). Recent developments in bamboo fiber-based composites: A review. Polymer bulletin, 76(5), 2655-2682.

Oerlemans, S., Sijtsma, P., \& López, B. M. (2007). Location and quantification of noise sources on a wind turbine. Journal of sound and vibration, 299(4-5), 869-883.

Qin, L., Wen-Ji, Y., \& Yang-lun, Y. (2012). Research on properties of reconstituted bamboo lumber made by thermo-treated bamboo bundle curtains. Forest Products Journal, 62(78), 545-550.

Rao, M. M. (2009). K., Mohana Rao, K., and Ratna Prasad, AV (2010)." Fabrication and testing of natural fibre composites: Vakka, sisal, bamboo and banana,". Materials \& Design, 31(1), 508-513.

Shao, Z., \& Wang, F. (2018). The Fracture Mechanics of Plant Materials (pp. 125-146). Springer Singapore, Singapore.

Takagi, H., \& Fujii, T. (2014). Mechanical characterization of bamboo fiber-reinforced green composites. In Key Engineering Materials (Vol. 577, pp. 81-84). Trans Tech Publications Ltd.

Vasudha, M., Harshal, P., Joshuva, A., \& Sugumaran, V. (2018). Effect of sampling frequency and sample length on fault diagnosis of wind turbine blade. Pakistan Journal of Biotechnology, 15, 14-17.

Widiastuti, I. (2016). Bamboo Laminated Composites for Wind Turbine Blade Material: a Review. In Prosiding Seminar Nasional UNS Vocational Day (Vol. 1).

Zhang, X., Fu, J., Paulo, A. C., Yu, C., \& Guebitz, G. M. (2012). Bioprocessing of bamboo materials. 